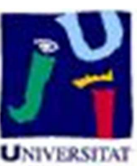

Título artículo / Títol article: Reaching for the Unreachable:

Reorganization of Reaching with Walking

Autores / Autors

Beata Joanna Grzyb, L.B. Smith, A.P. Del

Pobil

Revista:

Autonomous Mental Development, IEEE

Transactions on

Versión / Versió:

Versió post-print

Cita bibliográfica / Cita bibliogràfica (ISO 690):

GRZYB, B.; SMITH, L.; DEL POBIL, A.

Reaching for the unreachable:

reorganization of reaching with walking., Autonomous Mental Development, IEEE

Transactions on, 2013, vol. 5, no 2, p. 162-

172.

url Repositori UJI: 


\title{
Reaching for the unreachable: reorganization of reaching with walking
}

\author{
Beata J. Grzyb, Linda B. Smith, and Angel P. del Pobil, Member, IEEE
}

\begin{abstract}
Previous research suggests that reaching and walking behaviors may be linked developmentally as reaching changes at the onset of walking. Here we report new evidence on an apparent loss of the distinction between the reachable and nonreachable distances as children start walking. The experiment compared non-walkers, walkers with help, and independent walkers in a reaching task to targets at varying distances. Reaching attempts, contact, leaning, and communication behaviors were recorded. Most of the children reached for the unreachable objects the first time it was presented. Non-walkers, however, reached less on the subsequent trials showing clear adjustment of their reaching decisions with the failures. On the contrary, walkers consistently attempted reaches to targets at unreachable distances. We suggest that these reaching errors may result from inappropriate integration of reaching and locomotor actions, attention control and near/far visual space. We propose a rewardmediated model implemented on a NAO humanoid robot that replicates the main results from our study showing an increase in reaching attempts to nonreachable distances after the onset of walking.
\end{abstract}

Index Terms-infant reaching, perceived reachability, reaching and walking, near and far space integration.

\section{INTRODUCTION}

$\mathbf{E}$ FFECTIVE interaction with the objects placed in the surrounding space requires an integrated representation of the body and of the space surrounding the body, that is near (peripersonal) and far (extrapersonal) space. Near space is behaviorally defined as the space within the handreaching distance and far space represents the area outside the hand-reaching distance. Thus, different types of actions are possible depending on the distance away from a given object. That is, objects within near space can easily be grasped and manipulated, whereas locomotor action is required to get close enough to reach out and grasp objects beyond this space [1], [2], [3]. Such an action-based distinction between near and far space seems to be at the heart of Piaget's theory of spatial cognition in the sensorimotor period, where near space is defined as the space calibrated by reach and far space as that calibrated by self-produced locomotion [4], [5]. Not all actions, however, are available to the infants from the beginning. Accordingly, a major question is how infants' perception of space changes, and, more specifically, how their

B. J. Grzyb and A. P. del Pobil are with the Department of Computer Science and Engineering, Jaume I University, Castellón de la Plana, 12071, Spain. A. P. del Pobil is also with the Department of Interaction Science, Sungkyunkwan University, Seoul, South Korea. e-mail:grzyb@uji.es.

L. B. Smith is with Indiana University, Department of Psychological and Brain Sciences, Indiana University, United States of America

Manuscript received April 19, 2005; revised January 11, 2007. assessment of the object reachability changes as their bodies and motor skills change [6], [7], [8].

During the first months infants are fairly immobile. Their attention and exploration are confined to the reachable space. At or rapidly after the onset of reaching (around 4 months) infants clearly distinguish reachable and non reachable distances as they systematically do not reach for objects that are too far for contact to be made [9], [10], [7]. This boundary between near and far space appears to be recalibrated as children gain greater control over posture and especially the trunk during reaching movements, stabilities that enable them to reach farther [11], [12], [13]. The calibration, however, may not always be in the direction of increased accuracy in what is reachable. For example, Atkinson [14] commented on 12month olds attempts to reach for passing cars out of a window in everyday life situations, albeit the observation has never been empirically studied. McKenzie et al. [15] investigated 8to 12-month olds attempts to reach to far distances. Although contact with the target at the greatest distance was extremely difficult and rarely attained, older infants, often persisted in their attempts. McKenzie et. al conjectured that these reaching attempts play rather a social function of indicating to an adult observer the wish to obtain a distant object. Some evidences, however, suggest that infants' perception of reachable space may change when infants become mobile.

Locomotor experience seems to be a crucial factor of developmental change. With the emergence of upright locomotion several components, such as posture, balance control, muscle strength, and motivation are known to change [16]. Locomotion experience, however, does not create new psychological skills ex nihilo, and might not be responsible for the origins of the phenomena, but it can boost some psychological skills to a much higher level [17]. For example, infants who are walking show greater memory flexibility [18], a better understanding of object permanence [19], [20], [21], and affective communication in the mother-infant relationship [22], [23], [24]. Independent walkers spend significantly more time interacting with toys and with their caregivers, and also make more vocalizations and more directed gestures compared to non-walkers [24]. Although the onset of walking leads to rapid improvements in many aspects, it also contributes to momentary instability and loss of integrity in many seemingly unrelated systems. Learning to walk affects infants' sitting posture by increasing the magnitude of distance-related sway properties [25], and infants' reaching behavior as some infants return to two-handed reaching behavior [26]. Here we report evidence on an apparent loss of the distinction between the reachable and nonreachable distances as children start walking. 
We propose as working hypothesis that the representation of space changes with the onset of walking. Near and far space are integrated with the reaching and locomotion actions to constitute a coherent space representation. Our hypothesis builds on Atkinson's suggestion that infants around 12 months may fail to integrate information processed in at least four visual motor-attentional systems, that is oculomotor systems for foveating, size and shape constancy for successful object recognition, locomotor programming to get to the target, and finally, reaching and grasping [14]. The results obtained from our behavioral study seem to be in line with this hypothesis.

The experimental studies with adults show that the processes of representing space are well-suited to the different properties of reaching and locomotor actions and can efficiently guide both actions (e.g. [3]). In one study, neglect patients showed more severe neglect in extrapersonal than in peripersonal space in a bisection task [2]. While performing the bisection task by walking through the aperture, however, the patients deviated largely to the right side of the space. Since the aperture exists in peripersonal space at the moment of crossing, the patients should not exhibit neglect. This may suggest that once extrapersonal space has been coded no remapping from extrapersonal to peripersonal space takes place during self-locomotion, at least for short, linear trajectories. Similar evidences provide studies on locomotor actions. The central nervous system begins adjusting the parameters of locomotor actions to properly interact with the object (e.g. the goal or obstacle), even though it exists in extrapersonal space. For instance, the adjustments of the length of the stride to step over an obstacle begins a few steps before stepping over it [27]. These results suggest that space in the distance is likely to be proactively represented to enable the CSN to control locomotor actions in a feedforward way.

We employ reward-mediated learning to simulate the developmental process of near and far space representation in older infants. The model is composed of two neural networks for near and far space representations. Following the actionbased distinction between near and far space, these spaces are calibrated by reaching and walking actions respectively. To simulate younger infants' behavior, that is infants that are not yet able to self-locomote, initially only the network for near space representation is trained. However, after the onset of walking both networks are trained to enable planning and coordination of the walking and reaching actions. Our results obtained with the NAO humanoid robot replicate the results from our experimental study with children, and suggest some possible future experiments for validating the predictions generated by the proposed model.

\section{BEHAVIORAL STUDY}

\section{A. Experimental methodology}

Ethics Statement. Parents of all child participants provided informed consent prior to the experiment. All experimental protocols were approved by the Indiana University Institutional Review Board. In particular, the committee approved the consent materials which were signed by the parents of the participating children and are on file at the University.
Samples. The participants were 24 (17 female and 7 male) 12-month olds $(M=12.0$, range $=10.9$ to 12.9$)$. All infants were healthy on the day of testing, and had no known developmental disorders. Infants were recruited from a working- and middleclass population in the US Midwest. Based on parental reports on walking ability, children were assigned to 3 groups: nonwalkers, walkers with help, and independent walkers.

Stimuli. The stimuli were balls on dowels. The dowels were marked for distance for presentation. Three different balls of different sizes and colors were used with the purpose of keeping the infants engaged. The diameters (and colors) of the balls were as follows: $6 \mathrm{~cm}$ (green), $8 \mathrm{~cm}$ (red), and 10 $\mathrm{cm}$ (yellow). Analyses showed no effect of ball sizes and thus this stimulus property was not considered further.

Procedure and Equipment. Figure 1 presents our experimental setup. Participants were seated in a modified baby car seat. The chair allowed infants to lean freely forward without a danger of falling. The chair was fixed on a wooden platform so that the infants could not touch the floor with their feet, and hence could not use the floor as a support during reaching. The chair was tilted backwards about 11 degrees, so that sitting upright required infants to control their trunk. The balls on dowels were presented through a colorful stimuli display like a puppet theater - that also separated the experimenter from the infants. The balls were presented at distances 30 , $37,47,60$, and $70 \mathrm{~cm}$ from the infant using markings on the dowel and a ring through which the dowel was inserted in the puppet theatre to measure the distance. The entire experimental session was recorded with two cameras. The first camera located directly above the theatre recorded a birds-eye view of infants reaching movements and provided a clear view of the moment of contact. The second camera was placed on the side and captured the side view of the child that was used to determine the infants' leaning angle.

Prior to the experiment, the parents were asked to complete the questionnaire about their child's prehension and locomotor skills and postural control (see Appendix). Subsequently, the infant was seated in the chair and secured by the safety belt. The parent was located on a side, but slightly behind the infant. This arrangement meant that any communications with the parent (for example to help in retrieving the object) were likely to include the easily discriminable behavior of turning to look at the parent. The experimenter kneeled behind the puppet theatre display and presented the ball through its opening. No explicit instruction was given to the child. The parent was instructed not to interfere during the experiment, and encourage the infant only if the infant was reluctant to reach when the ball was very close to the infant's hands. If that should happen, the parent was told to ask the child in a playful way "Can you get it?" at the same time pointing to the ball to draw the child's attention towards it. At any distance, if reaching did not occur within $5 \mathrm{sec}$ after a stimulus presentation, the experimenter shook the ball to draw infant's attention towards it. The trial ended when the infant attempted to make contact with the object, or after 30 seconds. There was no explicit reward or praise of any kind provided to the infant after the trial for any tested distance so as to avoid the reward of reaching movements and so as to limit communication 
attempts with the experimenter. Each ball was presented one at a time. The five distances tested were: $30,37,47,60,70 \mathrm{~cm}$. We defined a trial block as a sequence of 9 test trials $(30,37$, $70,37,47,37,60,47,30)$. The first and the last trial presented a ball at the closest distance so as to encourage attempts to reach. The balls in the middle of the sequence were presented in a pseudo-random order such that no distance was repeated on adjacent trials. The sequence of trials was repeated until infants tired of the task with a maximum of 4 repetitions or 36 total trials. The mean number of trials (and SDs) completed by the infants was 25.5 (3.80).

Coding and Analysis. Infant behaviors during each ball presentation were coded from the video into 4 mutually exclusive categories: no response, lean only, reach only, reach and lean. A no response behavior was defined as a lack of any arm or trunk movements in the direction of the stimulus. A lean only behavior was classified as a forward inclination not accompanied by an arm movement in the direction of the ball. A reach only behavior was defined as a movement of one or two arms in the direction of the ball, but not accompanied by the trunk movement. In addition, the infant was coded as making a communication gesture that could be conceived of asking for help in getting the object if the infant pointed to the object or if the infant reached to the object while looking back at the parent. A reaching attempt was encoded as successful (scored as 1) if hand contact was made with ball, and unsuccessful (scored as 0 ) otherwise. The hand that contacted the ball, left, right or both, was also noted. Infant's leaning angle was measured from the video records. The video was stopped at the moment when the leaning angle was the greatest prior to contact with the ball. Following the coding scheme of McKenzie et al. [15], infants received a score from 1 to 8 depending of the lean extent. A score of 1 indicated a lean less than or equal to $24^{\circ}, 2$ a lean between $25^{\circ}$ and $29^{\circ}$, 3 a lean between $30^{\circ}$ and $34^{\circ}$, and so on with 8 indicating a lean of $55^{\circ}$ or more. A second coder coded $25 \%$ of the trials, and agreement equaled $93 \%$ for all coding categories.

\section{B. Results}

Figure 2 shows the mean proportion of trials in which infants reached and leaned, reached only, or neither reached nor leaned. As is evident, infants often leaned when reaching to near distances, but walkers were much more likely to lean when reaching to far distances. Each infant's proportions of REACH+LEAN and REACH were submitted to a 3 (Group: non-walker, walker with help, independent walker) by 2 (Response: REACH+LEAN, REACH) by 5 (Distance) analysis of variance for a mixed design. The analysis yielded main effects of Distance $F(4,84)=18.99, p<.001$, and reliable interactions between Distance and Group, $F(8,84)=2.06$, $p<.05$ and significant interactions between Response and Distance $(F=30.10 ; p<.001)$. Walkers reached more, reaches with leans were more frequent than reaches without leans, and overall reaching declined with the distance of the ball. The analysis showed a significant contrast between Distance of 47 and $60 \mathrm{~cm}, F(1,21)=10.89, p<.003$, as well as significant contrast in interaction between Distance of 47

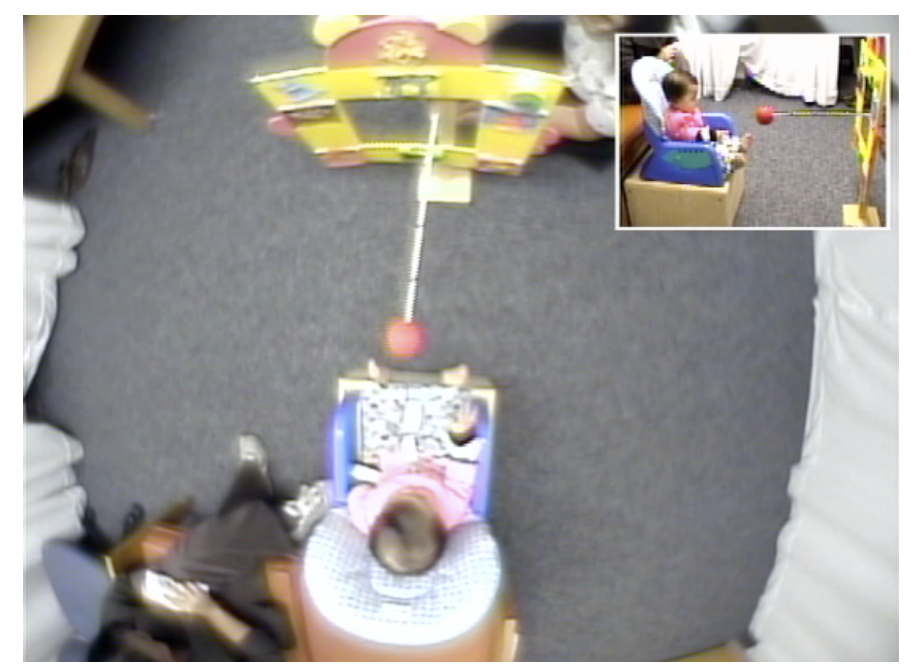

Fig. 1. Superimposed images from two cameras showing the experimental setup for the behavioral study. The first camera (large image) was located directly above the theatre, and recorded a birds-eye view of infants' reaching movements and provided a clear view of the moment of contact. The second camera (small image in the right corner) was placed on the side, captured the side view of the child, and was used to determine the infants' leaning angle.

$\mathrm{cm}$ and $60 \mathrm{~cm}$ and walking ability, $F(2,21)=3.84, p<.05$. While non-walkers reached less frequently at the Distance of $60 \mathrm{~cm}$, walkers (with or without help) continued reaching and leaning.

To further examine how walking experience changes the perception of reachable and non-reachable space, we calculated for each child the Attempted boundary defined as the longest distance at which the infant attempted to reach (all reaches whether leaning or not) on .50 or more of the trials. We defined the Contact boundary as the farthest distance at which the infant was able to make a contact on .50 or more of the trials. Figure 3 shows the histograms for the two measures. The modal Attempted boundary for walkers (with or without help) is the farthest and not reachable distance of $70 \mathrm{~cm}$, whereas the modal Attempted boundary for non-walkers is 47 , which is the same as the Contact boundary. However, a few nonwalkers perceived a distance as reachable at more than $47 \mathrm{~cm}$, which was much farther than the modal Contact boundary. This apparent shift in reaching boundary seen in some nonwalkers may be related to the experience in upright stance, as 2 out of 4 infants in the non-walker group that reached for far targets were able to stand without support for a few seconds.

Interestingly, an examination of reaches to far distances $(60 \mathrm{~cm}$ and $70 \mathrm{~cm})$ as a function of trial block reveals that all infants reached with high probability the first time the object was presented. The reaches of non-walkers, however, decreased over trial blocks showing a clear adjustment of reaching behavior at the "near boundary" distances in the task. Walkers in contrast persistently reached to both $60 \mathrm{~cm}$ and $70 \mathrm{~cm}$ distance regardless of the trial block showing little adjustment of their behavior with failures to make contact at the far distances. A 3 (Group: non-walker, walker with help, independent walker) by 3 (Trial block) mixed design analysis of variance yielded a significant group effect, $F(2,45)=4.08$, $p<.05$, and main effects of trial block, $F(2,90)=10.39$, 


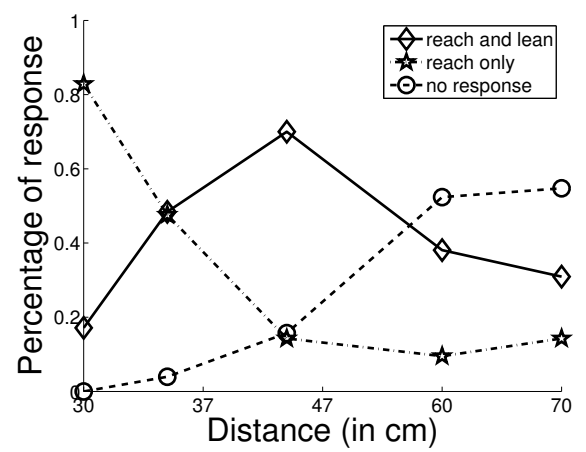

(a) Non-walkers

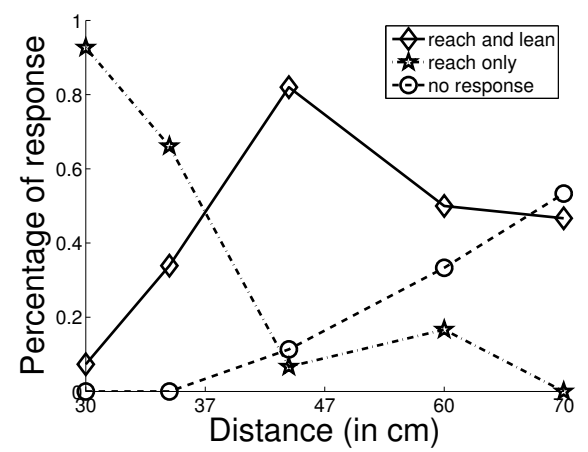

(b) Walkers with help

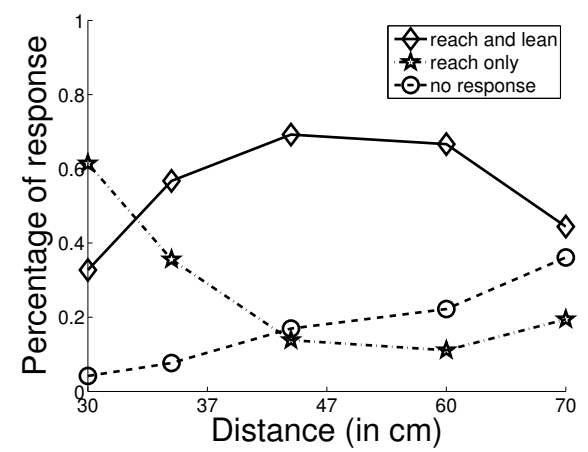

(c) Independent walkers

Fig. 2. Mean proportion of trials in which 12 month olds reached only, reached and leaned, or neither reached nor leaned. Leaning without reaching virtually never occurred and so is not shown.

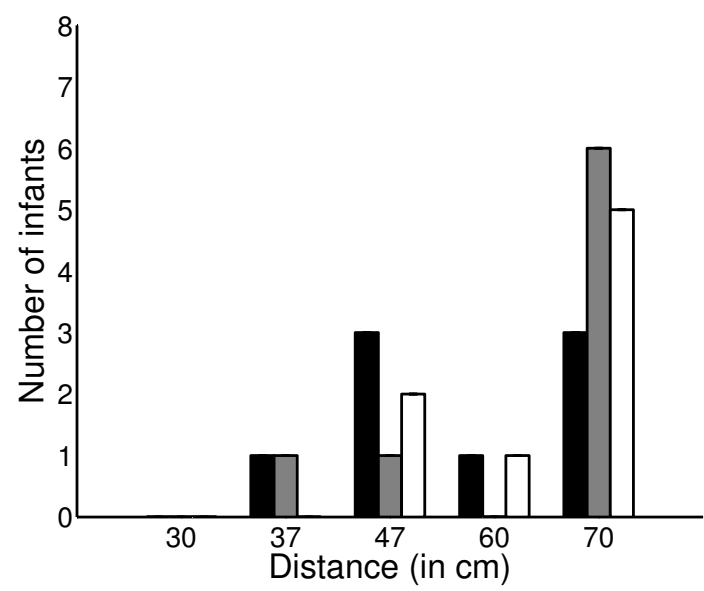

(a) The Attempted boundary

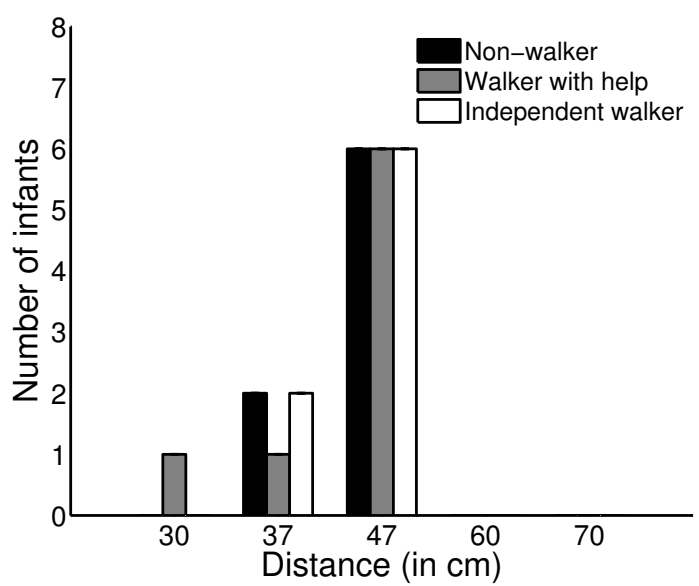

(b) The Contact boundary

Fig. 3. The Attempted boundary, that is the longest distance at infants attempted to reach on .50 or more of the trials and the Contact boundary, that is the farthest distance at which contacts are made on .50 or more of the trials for infants.

$p<.001$. Post hoc Tukey tests revealed a significant difference between the group of non-walkers and walkers with help $(p<0.05)$. Infants with walking experience in our experiment showed no evidence of learning from unsuccessful reaching trials.

Were these reaches to the ball at far distances by the 12 month olds not really acts of reaching but attempts to communicate? Although we can not unambiguously rule out this possibility, explicit attempts to communicate or ask for help were rare - vocal, gesture or looks at parents occurred on $14.21 \%$ of trials $(28 / 197)$ for non-walkers, on $5.56 \%(12 / 216)$ for walkers with help, and on $7.54 \%$ of trials (15/199) for walkers. These attempts were unrelated to distance for nonwalkers $(P=0.091)$ and walkers with help $(P=0.239)$, but moderately related to distance for independent walkers $(P=0.647)$. There is no converging evidence to support the idea that infants' reaches to far distances are not reaches but attempts to communicate or to ask for help in getting the ball.

All in all, the results of our experiment show that walkers constantly reached for the nonreachable targets showing little adjustment of their reaching with the failures of attempted reaches. We propose as working hypothesis that the representation of near space changes with the onset of walking.
The distance at which an object is perceived as reachable or not reachable may be determined in relation to a more global body schema. This schema can be defined as "an intermodal sense of the body, perceived by the infant as a functional whole situated in the environment and endowed with particular capacities for action" [13]. With the advent of upright locomotion, infants' perception of their own body capacities changes, as well as their representation of near space. When infants stand up and begin to walk, they have an expanded outlook over the space ahead. They see things from a different perspective - with more of a bird's-eye view. Walkers' hands are freed from supporting their bodies, and they can carry objects from place to place. For reaching and grasping the infant only needs a spatial representation of space which is relatively near to the body. For independent walking both peripheral vision and spatial layout some distance from the child must be represented to allow the child to find and retrieve objects in spatial locations that are beyond arm's length [14]. Walking provides necessary experience for proper integration of reaching and locomotor actions, attention control and near/far visual space. Novice walkers may reach more to distant objects because these objects would normally be "reachable by walking". 


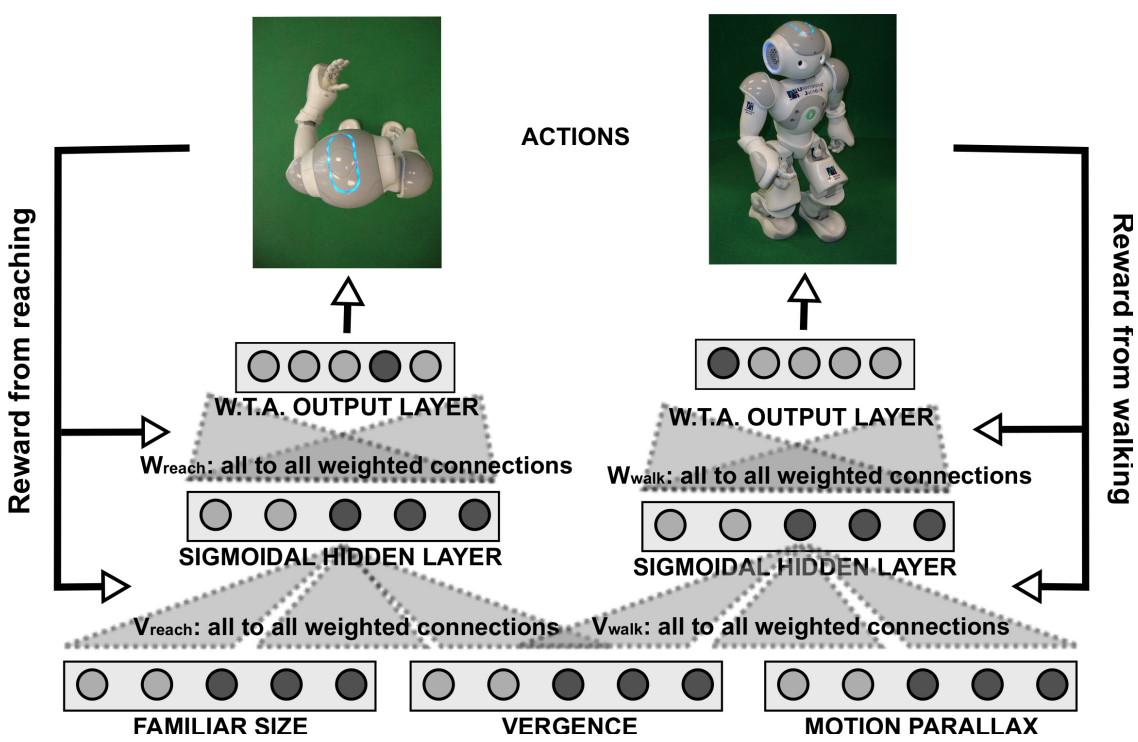

Fig. 4. General scheme of our reward-based learning architecture for near and far space representation. Two separate three-layer neural networks are used to approximate the state-action mapping function. The state here is depth-cue information, whereas action is equivalent to a specific depth estimate that can be associated with reaching and/or walking action.

\section{COMPUTATIONAL MODEL}

A reward-based learning approach has been shown to successfully mimic the development of near-optimal integration of visual and auditory cues in infants [28], [29], as well as to simulate the development of vergence eye movements [30] showing that even early sensory representations must be understood from the perspective of the behaving, goal-driven organism. A similar approach is taken here.

Figure 4 presents the outline of our architecture. The model is composed of two neural networks, one for near and one for far space representation. Since the architectures of the networks and existing connections between layers are similar, they will be discussed together and differences will be highlighted when necessary.

A three-layer neural network is used to approximate the state-action mapping function. The state here is an observed discretized distance to the target object provided by various depth cues. The action state discretized into 18 units represents different distances to reach or walk to the target object. More specifically, the input layer consists of $i=c * n$ binary neurons that encode the estimates of the $c$ (here $c=1 . .3$ ) different depth cues covering the $n$ (in our case $n=1 . .18$ ) discretized distance units within the range of $13 \mathrm{~cm}$ to $30 \mathrm{~cm}$. The activity of the neurons $x_{i}$ is one at depth estimated by the corresponding cue, otherwise zero.

The input neurons are all-to-all connected with weights $v_{i, j}$ to $j$ (here $j=1 . .18$ ) neurons in the hidden layer. A sigmoidal transfer function on the sum of the weighted inputs gives the outputs $y_{j}$ of the hidden neurons:

$$
y_{j}=\frac{1}{1+e^{-\sum_{i} v_{i, j} x_{i}}}
$$

The hidden neurons are fully connected to output neurons $k$ with weights $w_{j, k}$. All weights are drawn from uniform distributions, $v_{i, j}$ between -0.1 and 0.1 , and $w_{j, k}$ between -0.1 and 0.1 .

Each output unit represents an action. Representation of an action differs in both networks. While distances in the network for near space are represented in centimeters, distances in the network for far space are represented in steps. In case of the network for near space $k_{r}\left(k_{r}=1 . .18\right)$ reaching actions are possible and the binning size, that is the parameter responsible for discretization of the action space is set to $1 \mathrm{~cm}$. In case of the far space representation, $k_{w}\left(k_{w}=1 . .4\right)$ walking actions are possible, and the binning size is set to 1 step.

The activation of the output neurons $z_{k}$ is given by the weighted sum of the hidden layer activity, representing an approximation of the appropriate Q-value. Based on the network's outputs, one action is chosen according to the softmax action selection rule [31]:

$$
P_{t}(k)=\frac{e^{Q_{t}(k) / \tau}}{\sum_{b=1}^{n} e^{Q_{t}(b) / \tau}}
$$

where $P_{t}(k)$ is the probability of selecting an action $k, Q_{t}(k)$ is a value function for an action $k$, and $\tau$ is a positive parameter called temperature that controls the stochasticity of a decision. A high value of $\tau$ allows for more explorative behavior, whereas low value of $\tau$ favors more exploitative behavior. We start with a high temperature parameter $\tau=\tau_{0}$, so that the selection of action is only weakly influenced by the initial reward expectations. In our experiments, $\tau$ decreases exponentially with time $\tau(t)=\tau_{0}^{\left(\frac{v_{\tau}-t}{v_{\tau}}\right)}$, where $\tau_{0}=10$ and $v_{\tau}=15000$ in case of the network for reaching and $v_{\tau}=5000$ in the other case.

After performing the selected action $\hat{k}$ the true reward $r(\hat{k})$ is provided. The reward is maximal when $\hat{k}$ equals the true object position $k_{t}$, decaying quadratically with increasing distance within a surrounding area with radius $\rho$ (here $\rho=4$ 
in case of near space representation and $\rho=0$ in other case).

$$
r(\hat{k} \mid X)=\max \left(0,\left(\rho-\left|\hat{k}-k_{t}\right|\right)\right)^{2}
$$

To minimize the error between the actual and expected reward, we make use of the gradient descent method which is widely used for function approximation, and is particularly well suited for reinforcement learning:

$$
\begin{gathered}
v_{i, j}(t+1)=v_{i, j}(t)-\epsilon\left(r_{\hat{k}}-z_{\hat{k}}\right)\left(-w_{j, \hat{k}}\right) y_{j}\left(1-y_{j}\right) x_{i} \\
w_{j, \hat{k}}(t+1)=w_{j, \hat{k}}(t)-\epsilon\left(r_{\hat{k}}-z_{\hat{k}}\right)\left(-y_{j}\right)
\end{gathered}
$$

In case of the update of weights $w_{j, k}$ only the output weights connected to the winning output unit $\hat{k}$ are updated. The learning rate $\epsilon$, decreases exponentially, according to the formula $\epsilon(t)=\frac{\epsilon_{0}}{\operatorname{ceil}\left(\frac{t}{v_{\epsilon}}\right)}$, where $\epsilon_{0}=0.05$ (for both networks), and $v_{\epsilon}=15000$ in case of the network for reaching, and $v_{\epsilon}=200$ in other case.

One of the shortcomings of the reward-based methods is the large number of training examples needed for the neural network to converge. For example, to train the network for reaching we need approximately $t=15000$ time steps. Such a large number of repetitions would be extremely timeconsuming and unfeasible for any robotic platform. Therefore, the initial weights of the neural networks are trained offline using real data collected with the use of the NAO humanoid robot as explained below. Such a neural network is subsequently implemented on our robotic setup and its performance evaluated with real-time data.

\section{Robot Study}

\section{A. Experimental framework and software architecture}

Robotic Setup: Aldebaran's commercially available humanoid robot NAO with $25 \mathrm{DoF}$ is used as a validation platform for the proposed working hypothesis. The robot is provided with two identical video cameras, one placed in the forehead and the other in the chin. These locations, however, do not allow the use of stereo vision methods for depth calculation. Additionally, the NAO robot is also equipped with 3 -axis linear accelerometers that are used to measure travelled distance.

Training data collection: The visual depth cue data, such as familiar size, vergence and motion parallax are collected with the use of the NAO humanoid robot. The procedure is as follows. The object is placed manually in front of the robot approximately on its eye-height. The robot centers the objects in the image, and then it estimates the distance to the object using static depth estimation methods (i.e. familiar size, see Appendix). Afterwards, it executes a lateral movement to the right during $2.4 \mathrm{~s}$. During the movement the robot accesses the acceleration data with a constant sample period $\Delta T$, and calculates its displacement according to the state updating of eq. (8) (See Appendix), where $\Delta p$ and $v$ at the starting time $\left(\Delta p_{0}, v_{0}\right)$ were both set to zero. Once the final position is reached, the object distance is calculated again by using the motion parallax method. Then, the image is once again centered so as to calculate the distance by motion perspective. After all distance methods have been calculated, the object is replaced manually for the next trial. The measures are taken every $1 \mathrm{~cm}$.

Reaching behavior in the NAO humanoid robot: Within our framework, we provided the NAO robot with a reaching module. The spatial position of an object is maintained by two implicit frames of references, one head-centered and one arm-centered (for more details refer to [32], [33]). The headcentered frame of reference provides the object location in terms of gaze direction (pan, tilt), together with its distance. The arm-centered frame of reference is defined by the angular position of the joints (shoulder pitch, shoulder roll and elbow), that allow the robot to reach and grasp the object. Both the direct and the inverse mapping between the two coordinate systems are encoded by two radial basis functions networks. The hidden layer of the two networks is composed by 343 neurons with Gaussian activation, those centers are uniformly distributed on a $7 \times 7 \times 7$ lattice of the input space. The output is computed as the weighted sum of the hidden layer, where the weights are learned by means of the least square algorithm.

Distance information used to encode the spatial position of the object in the head-centered frame of reference can be replaced with the output of another computation as long as it provides neural activation which is related with the distance of the target. As distance here we use the reaching distance provided by the computations of the model presented in the previous section. In other words, various visual depth cues (e.g. familiar size, vergence, motion parallax) are first integrated in a spirit of the Bayesian theorem using the model described in the previous section. The outcome of such computation, together with the the gaze direction, is subsequently fed to the reaching module, which outputs the angular positions of the joints that allow the robot to reach for a given target.

\section{B. Robot Experiments}

As in our behavior study with infants, the main objective of the robot is to decide whether to reach or not for an object presented at one of the five distances. Since the NAO robot is much smaller than an average 12-month-old infant, we adjusted the testing distances to reflect its size. Five different distances are tested, 2 near distances that easily allow the robot for reaching and grasping the objects $(13 \mathrm{~cm}$ and $15 \mathrm{~cm})$, one distance on the border of reachable space $(21 \mathrm{~cm})$, and two distances clearly outside of the reachable space $(27 \mathrm{~cm}$ and $29 \mathrm{~cm}$ ). Each test trial is repeated 10 times.

\section{Experiment 1: Before the onset of walking}

The task of the learner is to estimate the reaching distance to a target object. The learner obtains noisy estimates of depth and based on its internal predictions reaches towards the object. We employ here a kinematics model of the arm to test the outcome of reaching movement. Based on the accuracy of the reaching distance, the learner obtains a varying amount of reward and learns to predict the amount of expected reward when performing each action in a given situation. The learner represents its reward estimates for particular state and action pairs as so called Q-values [31]. In our 


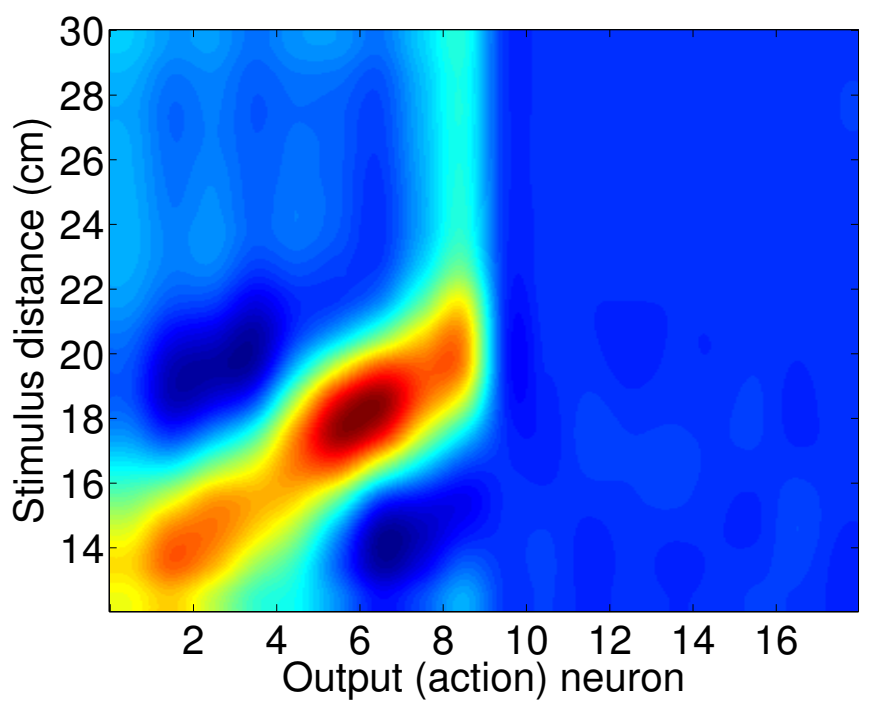

(a) Before walking

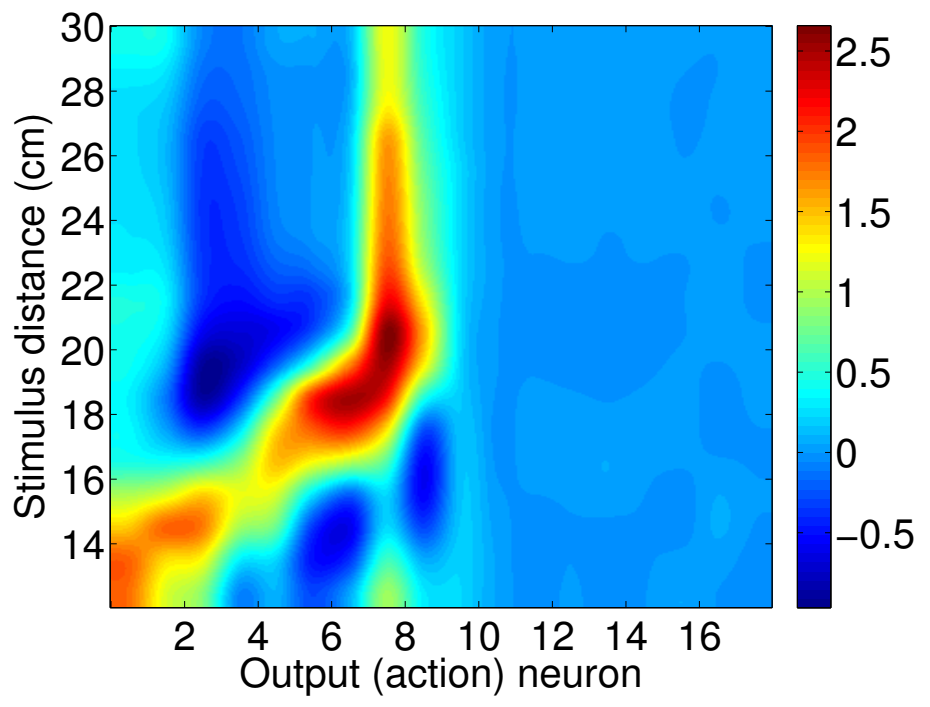

(b) After walking

Fig. 5. Examples of learned Q-value functions for near space representation network. A colormap shows reward predictions (Q-values) of the output neurons ( $\mathrm{x}$-axis, $\mathrm{n}=18$ neurons) for all distances used during training (y-axis). Figure on the right shows how the representation of near space has changed after the onset of walking; the output neurons that previously encoded the distances near the boundary of reachable space, activate now also for distances outside of the reachable space.

implementation, the Q-function is approximated by a neural network (as described in Sec. III). Depending on these reward expectations, the learner probabilistically chooses an action using a softmax function. Training of the network starts with a high temperature parameter $\tau$, so that the selection of action is only weakly influenced by the initial reward expectations. A gradient descent learning algorithm is used to change the synaptic weights of the network so that the reward prediction of the winning action reflects better the difference between predicted and obtained reward.

The activations of the output neurons represent reward predictions that can be used to discriminate near and far space. A high value of reward prediction corresponds to near and easily reachable distances, whereas a low value to nonreachable distances. Figure 5a shows an example of a learned Q-value function. Initial weights of the network are set to small random values, and thus implicate small reward expectations. During the learning process, only the weights of the neurons that encode near distances are rewarded leading slowly to the emergence of the representation of near space. The linear trend suggests that each neuron achieves maximum level of activation for each of the reachable distances. Furthermore, only approximately half of the neurons encode near space.

Such a neural network trained in simulation was subsequently used to test the robot's performance under realworld conditions. Figure 6a shows mean reaching attempts of the robot compared to the mean reaching attempts of 12month-old non-walkers. Both the infants and the robot reached to the easily accessible distances. Their reaching attempts, however, decreased with the increasing distance showing a clear boundary between reachable and nonreachable distances. The border of the reachable space in case of the robot study seems to be more abrupt than in infants' study. It's worth noting here that reaching is a complex motor skill and many factors - other than space perception - may influence infants' reaching decisions, such as for example curiosity.

\section{Experiment 2: After the onset of walking}

The task of the learner is to estimate reaching and/or walking distance to a target object based on the input noisy estimates of distance and internal predictions of rewards. If the object is placed beyond the reachable space the model estimates the necessary number of steps for walking and the remaining distance for reaching. Thus, the use of two separate networks enables planning and coordination of reaching and walking actions. Initially, the weights of far space representation are set to small random values, whereas the weights of near space representation are kept unchanged from the previous training. The weights of both networks are updated using a gradient descent learning algorithm. The learner obtains a varying amount of reward depending on the accuracy of estimation and learns to predict the amount of expected reward when performing each action in a given situation. Rewards for the reaching module are proportional to the effort (here a number of steps) required to get close enough to the object to enable reaching. Based on reward expectations, the learner probabilistically chooses appropriate actions using corresponding softmax functions. The training of the network for far space representation begins with a high value of temperature, so that the selection of action is only weakly influenced by the initial reward expectations. On the contrary, in case of near space representation the value of temperature is kept low, and reward expectations strongly influence action selection.

Figure 5b shows how the representation of near space has changed after the onset of walking. The output neurons that previously encoded the distances near the boundary of reachable space, activate now also for distances outside of 


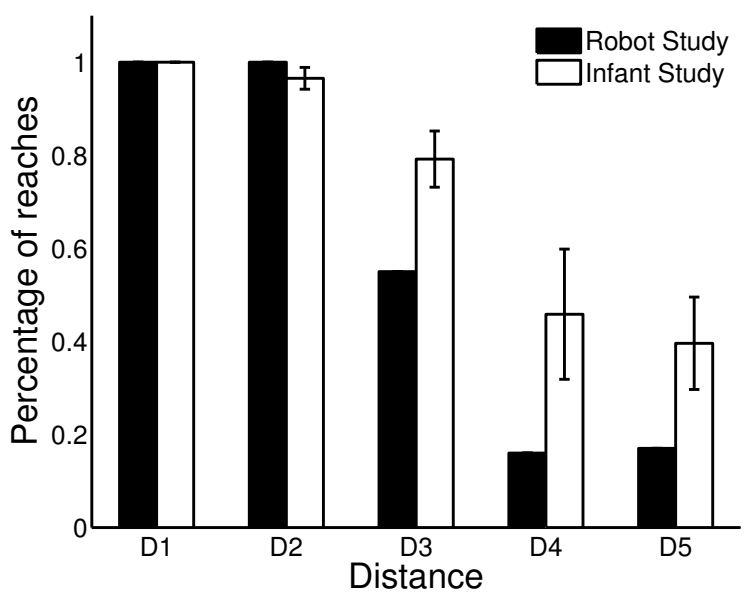

(a) Non-walkers

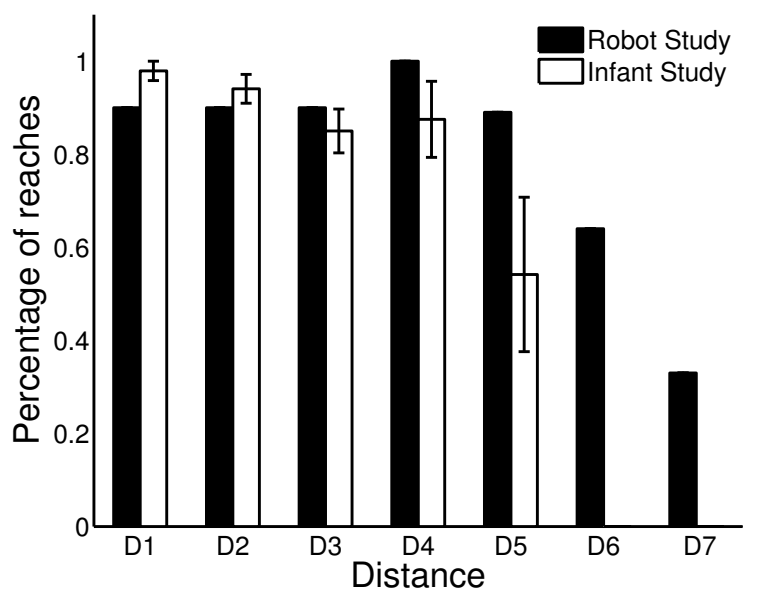

(b) Walkers

Fig. 6. Percent of reaches with standard deviation (range of error bars) for infants with non-walkers (on the left) and independent walkers (on the right) to 5 different distances $(30,37,47,60,70 \mathrm{~cm})$. The computational model was tested with the use of the NAO humanoid robot, and the results are provided here for comparison. Since the NAO robot is much smaller than an average 12-month-old infant, testing distances were adjusted accordingly (13, 15, 21, 27, 29 $\mathrm{cm})$. Additionally, we tested the robot with two far distances (37 and $39 \mathrm{~cm}$ ).

the reachable space. The activation is stronger for distances slightly outside of the boundary and decreases with the increasing distance. This may suggest that the perceived boundary of reachable space has been shifted, and infants would not attempt to reach for the objects beyond that newly established boundary.

The performance of the neural network trained in simulation is evaluated with the use of the NAO humanoid robot. Since the infants in our experiment were sitting in a chair with a seatbelt fastened, they were not able to stand up and walk towards the presented targets. Similarly, in the robot experiment we disabled the execution of walking. Figure $6 \mathrm{~b}$ shows the mean reaching attempts of the robot. The representation of near space has changed, and the distances previously represented as unreachable now are viewed as reachable. However, when the execution of walking was enabled, the robot in all cases was able to walk towards and then successfully reach and grasp the object using distance information provided by the networks. Additionally, we presented the robot with two very far distances to see if the reaching attempts rate would decrease. Indeed, our results seem to confirm this hypothesis suggesting at the same time that far distances used in our behavioral study could not be far enough to inhibit infants with standing experience from reaching.

\section{DiscusSION}

Our main goal was to establish whether infants with walking experience would reach more for nonreachable objects than infants without such an experience. Our findings were consistent with our predictions. That is, walkers (with or without help) constantly reached for nonreachable targets showing little adjustment of their reaching behavior with the failures of attempted reaches.

The results obtained from our empirical study seem to be in line with Corbetta \& Bojczyk's [26] suggestion that walking and reaching systems may be linked developmentally as reaching reorganizes at the onset of walking. The increased reaching to nonreachable objects in recent walkers provides further evidence that development is not necessarily continuous, and that once learned skills are not invulnerable to changes. We proposed as working hypothesis that the representation of space, not only far space, but also near space changes with the onset of locomotion so that the newly developing representation of the far space can be integrated into a coherent space representation. This new space representation arises out of high behavioral instability - a notion that is compatible with dynamical systems theory [34].

Once infants are able to maintain the upright posture and make first independent steps, the whole body becomes part of the reaching movement. Standing upright increases the potentially relevant degrees of freedom; a small step and a slight bend of the trunk are readily incorporated into the arm movement [35]. Adults overestimate the maximum distance at which objects can be contacted when utilizing just one degree of freedom [36]. This may suggest that reachability judgements are based on a mentally simulated reach that includes all degrees of freedom that are normally available to solve this task [37], [36]. Thus, the reachability judgements are determined in relation to a more global body schema. As mentioned before, infants' perception of their own body capacities changes with the advent of upright locomotion. It is plausible that novice walkers may reach more frequently to nonreachable objects because they are not yet able to mentally immobilize the body's remaining degrees of freedom. This would imply that attempted reaches should decrease with increasing distance of the object. Indeed, we could observe a small decrease in infants' reaching attempts at $70 \mathrm{~cm}$ comparing to $60 \mathrm{~cm}$ distance. Many infants, however, still attempted to reach at the farthest distance of $70 \mathrm{~cm}$. It may be that the farthest distance in our experiment was not far enough to inhibit infants with standing experience from reaching. 
What would happen if the objects were presented $100 \mathrm{~cm}$ away or more? According to our model, reaching attempts would decrease with increasing distance. Further empirical studies with children are needed to test this prediction.

With our reward-mediated learning model we replicated the main results from our empirical study showing an increase in reaching attempts to nonreachable distances after the onset of walking. Our secondary finding was that all infants reached to out-of-reach objects the first time they were presented, but only non-walkers were able to adjust their reaching decisions to the failures in attempted reaches. The presented model, however, can not be used to investigate the reasons behind these momentary changes in non-walkers' behavior.

Borrowing from Thelen and Ulrich's analysis of the development of infant walking, a decision to reach may well depend on many cognitive, motivational, social, perceptual and motor factors [38]. Developmental changes in any or several of these components could be central to the present findings. The presented mechanism may be just one possible explanation of the observed distance errors.

Previously, we examined how changes in motivation may influence infants' reaching decisions [39]. More specifically, we suggested that infants' perception of distance changes in a new upright posture. Infants, however, fail to correctly update the boundaries of their reachable space because of their decreased ability to learn from the errors. This may result from infants' high level of sense of control upon making their first steps. Omission of the errors was suggested to enable selection of different behaviors in a context when they normally would not be selected providing more learning opportunities for finetuning these behaviors. For example, ignoring the errors in reaching may be helpful in fine-tuning the newly acquired walking skill since a primary motive for walking is to reach for something.

Furthermore, we investigated how reorganization of the processes responsible for integration of different visual depth cues at the onset of walking may influence infants reaching behavior. Our hypothesis was based on the suggestion that attention to environment is one of the critical mechanisms by which developmental changes occur [17]. The ability to selflocomote lures attention to far space, especially to the location toward which the infant is moving. Such a reallocation of attention from near to far space modifies the use of various sources of depth information and leads to a change in the use of monocular static information specifying depth relations. We proposed that a shift of attention from near to far space causes a reorganization of various visual depth-specifying cues, and walking promotes re-calibration of distance perception according to the new motoric metric. This hypothesis was tested with the reward-mediated learning model [40]. The results of simulations showed an increase in the near-far confusions during the learning of far space representation, similarly as in the case of recent walkers.

Contrary to this model, the one presented in this paper processes the information about near and far distances in two separate maps, similarly as is done in humans and primates. Such a division enables a subject (or a robot) to calibrate near and far space according to different metrics.
For example, the input information about object distance as well as the output information about the reaching distance was in centimeters, whereas the output information about walking distance was in steps. Such an architecture has a potential to entirely eliminate the necessity of representing the input information about distance in any explicit metric system. Any input/output information would be acceptable, as far as it provides information that is somehow correlated with distance. For example, familiar size information in our framework could be represented by a relation of retinal size to memorized haptic object size. In this way, the robot using only its senses would be able to implicitly map the surrounding space and calibrate according to its body size and action capabilities. Another important advantage of dual network representation is that planning an action in far space is possible, but requires proper coordination of the near and far maps in time. The reintegration of various depth cues at the onset of walking behavior, however, has not been tackled in the present work.

The three proposed explanations of infant behavior may not be mutually exclusive and may also overlap in underlying mechanisms. Decreased learning from errors may be related to a change in the threshold for activating the reaching action. Such a lower threshold may be a motivational force that drives the system to collect more information for appropriate integration of visual depth cues and calibration of absolute distance perception according to the new motoric factors. Similarly, it also provides necessary experience for learning how to plan and coordinate reaching and walking actions in space. The future work should focus on integration of these models for better understanding of the complex mechanisms underlying these goal-directed actions. On the other hand, further investigation should focus on how a change in perceived object reachability may be related to other changes in infant behavior that happen about the same time (e.g. object manipulation, search for hidden objects, ability to imitate actions requiring spatial relations etc.). The new findings from such behavioral studies would be helpful in refining and developing in more detail our reward-mediated model for simulating an actionbased space and distance perception in infants.

\section{Conclusions}

The main contribution of our behavioral study is the finding that the alignment of attempted and reachable distances changes at the onset of walking. We proposed that these reaching errors may result from inappropriate integration of reaching and locomotor actions, attention control and near/far visual space. With our reward-mediated framework, we replicated the main results from our empirical study and generated new predictions. According to our tests, infants would not reach for objects located much farther than in our empirical study, and they would actually walk to reach and grasp far objects if they were not fastened by the seatbelt. Further empirical studies with children are necessary to validate these predictions. 


\section{APPENDIX}

\section{PARENTAL QUESTIONNAIRE}

Functional assessment of motor functions adapted from [41].

1) Grasping:

- infant is able to grasp and hold only one object

- infant is able to grasp and hold two objects

- infant is able to grasp and hold three objects

2) Type of grasping:

- infant uses only the palmar grasp

- infant is able to use the radial palmar grasp

- infant is able to use the scissor grasp

- infant is able to use the inferior scissor grasp

- infant is able to use the pincer grasp

3) Sitting:

- infant is unable to sit without support

- infant can sit without support for a few seconds

- infant is able to sit without support for more than $10 \mathrm{~s}$

4) Standing:

- infant is unable to stand without support

- infant can stand without support for a few seconds

- infant is able to stand without support for more than $10 \mathrm{~s}$

5) Side step:

- when pushed laterally when held in a standing position, the infant does not make a step sidewards

- when pushed laterally when held in a standing position, the infant performs a sidewards step

6) Walking:

- infant is unable to walk without support

- infant can walk with help

\section{APPENDIX}

\section{DEPTH ESTIMATION METHODS}

Accurate estimation of depth is a challenging issue in the field of computer vision. Since three dimensional real objects present in the environment are projected into the two dimensional surface of the camera sensor the depth information is lost, and additional constraints are required for its extraction. In this work, the following methods for depth estimation are used.

Familiar Size. When a physical size of an object is known, its absolute depth can be calculated by using the following equation:

$$
z_{f s}=f \cdot \frac{S_{\text {physical }}}{S_{\text {observed }}}
$$

where $f$ is the focal length (in pixel term), $S_{\text {physical }}$ and $S_{\text {observed }}$ are a physical (in meters) and an observed size (in pixel term) of a known feature, respectively. Equation (6) assumes that the observed feature is presented orthogonally to the camera sensor. In case such a condition is not fulfilled and no information about inclination of the object is provided, the depth estimation can be largely overestimated.
Parallax. Extracting the depth information from the parallax is straightforward once the displacement of the camera $\Delta p$ and the displacement of the object in the image $\Delta x$ are known using equation (7):

$$
z_{p x}=f \cdot \frac{\Delta p}{\Delta x}
$$

where $\Delta p$ is the displacement of the camera. The displacement of the camera can be provided by an inertial sensor. In the case we can access the instantaneous acceleration $a_{k}$ with a constant sample period $\Delta T$ and by assuming that the acceleration is constant during such a period, it is possible to calculate $\Delta p$ using the following discrete system:

$$
\left[\begin{array}{c}
\Delta p_{k+1} \\
v_{k+1} \\
a_{k+1}
\end{array}\right]=\left[\begin{array}{ccc}
1 & \Delta T & \Delta T^{2} / 2 \\
0 & 1 & \Delta T \\
0 & 0 & 1
\end{array}\right] \cdot\left[\begin{array}{c}
\Delta p_{k} \\
v_{k} \\
a_{k}
\end{array}\right]
$$

where $v_{k}$ is the velocity and the only observable variable at the time $k$ is the acceleration $a_{k}$. The problem of this approach is that is a double integrator and a bias in the measure, for example due to the change of the temperature, can lead to an unbounded estimation error.

Motion perspective. The motion perspective allows for calculating the depth of a feature using a technique that is a mix between the stereopsis and the parallax. As for the parallax the robot moves perpendicularly to the optical axis, while the yaw motor of the neck is rotated to maintain the fixation as for the stereopsis. Assuming that at the beginning the object is in front of the robot, so the neck is in the position zero, the depth can be estimated as follows:

$$
z_{t r}=\frac{\Delta p}{\tan (\theta)}
$$

where $\theta$ is the angular position of the neck necessary for gazing the target point after the displacement $\Delta p$. The displacement can be computed as for the parallax (see equation $8)$.

\section{ACKNOWLEDGMENTS}

This research was partly supported by the WCU Program of the National Research Foundation of Korea funded by the Ministry of Education, Science and Technology (Grant No. R312008-000-10062-0), by Ministerio de Ciencia e Innovación (FPU grant AP2007-02565, DPI2011-27846), by Generalitat Valenciana (PROMETEO/2009/052) by Fundació Caixa Castelló-Bancaixa (P1-1B2011-54), and also by the National Institute for Child Health and Development (R21HD068475) to the second author. The authors would like to thank Vicente Castelló for his help in implementing the model and performing robot experiments, and Charlotte Wozniak for her help in the preparation of the behavioral study and coding.

\section{REFERENCES}

[1] A. Berti and F. Frassinetti, "When far becomes near: remapping of space by tool use," Journal of Cognitive Neuroscience, vol. 12(3), pp. 415420,2000

[2] A. Berti, N. Smania, and A. Allport, "Coding of far and near space in neglect patients," Neuroimage. 2001 Jul;14(1 Pt 2):S98-102., vol. 14 pp. 98-102, 2001. 
[3] T. Higuchi, K. Imanaka, and A. E. Patla, "Action-oriented representation of peripersonal and extrapersonal space: Insights from manual and locomotor actions," Japanese Psychological Research, vol. 48(3), pp. 126-140, 2006.

[4] E. Lamb, L. Sherrod, L. Sherrod, and M. Lamb, Infant Social Cognition: Theoretical and Empirical Considerations. Taylor \& Francis, 1980. [Online]. Available: http://books.google.es/books?id=vtjJx_UBJIMC

[5] J. Piaget, The construction of reality in the child. New York: Basic Books, 1954.

[6] R. K. Clifton, P. Rochat, R. Y. Litovsky, and E. E. Perris, "Object representation guides infants' reaching in the dark," Journal of Experimental Psychology: Human Perception and Performance, vol. 17(2), pp. 323329, 1991.

[7] R. K. Clifton, F. E. Perris, and A. Bullinger, "Infants' perception of auditory space," Developmental Psychology, vol. 27, pp. 187-197, 1991.

[8] K. E. Adolph, "Specificity of learning: Why infants fall over a veritable cliff," Psychological Science, vol. 11(4), pp. 290-295, 2000.

[9] J. Field, "The adjustment of reaching behavior to object distance in early infancy," Child Development, vol. 47(1), pp. 304-308, 1976.

[10] — "Relation of young infants reaching behavior to stimulus distance and solidity," Developmental Psychology, vol. 12(5), pp. 444-448, 1976.

[11] A. Yonas and B. Hartman, "Perceiving the affordance of contact in fourand five-month-old infants," Child Development, vol. 64(1), pp. 298308, 1993.

[12] P. Rochat and N. Goubet, "Development of sitting and reaching in 5to 6-month-old infants," Infant Behavior and Development, vol. 18, pp. 53-68, 1995.

[13] P. Rochat, N. Goubet, and S. J. Senders, "To reach or not to reach? perception of body effectivities by young infants," Infant and Child Development, vol. 8, pp. 129-148, 1999.

[14] J. Atkinson, The developing visual brain. Oxford University Press Inc., New York, 2000.

[15] B. E. McKenzie, H. Skouteris, R. H. Day, B. Hartman, and A. Yonas, "Effective action by infants to contact objects by reaching and leaning," Child Development, vol. 64(2), pp. 415-429, 1993.

[16] E. Thelen, Motor development in children: Aspects of coordination and control. Dordrecht, The Netherlands: Martinus Nijhoff, 1986, ch. Development of coordinated movement: Implications for early human development, pp. 107-124.

[17] J. J. Campos, D. I. Anderson, M. A. Barbu-Roth, E. M. Hubbard, M. J. Hertenstein, and D. Witherington, "Travel broadens the mind," Infancy, vol. 1, pp. 149-219, 2000.

[18] J. Gross, S. Seehagen, and H. Hayne, "How long does motor status influence the flexibility of memory retrieval during infancy?" in 38th Annual Meeting of the International Society for Developmental Psychobiology, 2005.

[19] M. Bell and N. Fox, "The relations between frontal brain electrical activity and cognitive development during infancy," Child Development, vol. 63, pp. 1142-1164, 1992.

[20] B. I. Bertenthal and J. J. Campos, Advances in infancy research. Norwood, NJ: Ablex, 1990, ch. A systems approach to the organizing effects of self-produced locomotion during infancy, pp. 1-60.

[21] J. Gross, H. Hayne, N. Perkins, and B. McDonald, "Amount of crawling and walking experience has varying effects on cognitive development during infancy," in The annual meeting of the XVth Biennial International Conference on Infant Studies, 2006.

[22] Z. Biringen, R. N. . Emde, J. J. Campos, and M. I. Appelbaum, "Affective reorganization in the infant, the mother, and the dyad: the role of upright locomotion and its timing," Child Development, vol. 66(2), pp. 499-514, 1995.

[23] Z. Biringen, R. N. . Emde, J. J. Campos, and M. Appelbaum, "Development of autonomy: role of walking onset and its timing," Perceptual and Motor Skills, vol. 106, pp. 395-414, 2008.

[24] M. W. Clearfield, "Learning to walk changes infants' social interactions," Infant Behavior \& Development, vol. 34(1), p. 2011, 2011.

[25] L. Chen, J. S. Metcalfe, J. J. Jeka, and J. E. Clark, "Two steps forward and one back: Learning to walk affects infants' sitting posture," Infant Behavior and Development, vol. 30, pp. 16-25, 2007.

[26] D. Corbetta and K. E. Bojczyk, "Infants return to two-handed reaching when they are learning to walk," Journal of Motor Behavior, vol. 34(1), pp. 83-95, 2002.

[27] R. Moraes, M. A. Lewis, and A. E. Patla, "Strategies and determinants for selection of alternate foot placement during human locomotion," Experimental Brain Research, vol. 159(1), pp. 1-13, 2004.

[28] C. Karaoguz, T. H. Weisswange, T. Rodemann, B. Wrede, and C. A. Rothkopf, "Reward-based learning of optimal cue integration in audio and visual depth estimation," in The 15th International Conference on Advanced Robotics, Tallinn, Estonia, 2011.

[29] T. H. Weisswange, C. A. Rothkopf, T. Rodemann, and J. Triesch, "Bayesian cue integration as a developmental outcome of reward mediated learning," PLOS ONE, vol. 6(7), pp. 1-11, 2011.

[30] A. Franz and J. Triesch, "Emergence of disparity tuning during the development of vergence eye movements," in In International Conference on Development and Learning, 2007, pp. 31-36.

[31] R. S. Sutton and A. G. Barto, Reinforcement Learning: An Introduction. MIT Press, Cambridge, MA, 1998.

[32] E. Chinellato, M. Antonelli, B. Grzyb, and A. del Pobil, "Implicit sensorimotor mapping of the peripersonal space by gazing and reaching," IEEE Transactions on Autonomous Mental Development, vol. 3, no. 1, pp. 43-53, 2011.

[33] M. Antonelli, B. J. Grzyb, V. Castelló, and A. P. del Pobil, "Plastic representation of the reachable space for a humanoid robot," in From Animals to Animats 12, ser. Lecture Notes in Computer Science, T. Ziemke, C. Balkenius, and J. Hallam, Eds. Springer Berlin / Heidelberg, 2012, vol. 7426 , pp. $167-176$

[34] E. Thelen and L. Smith, A Dynamic Systems Approach to the Development of Cognition and Action. MIT Press, 1994.

[35] M. Flanders, L. Daghestani, and A. Berthoz, "Reaching beyond reach," Exp Brain Res, vol. 126, pp. 19-30, 1999.

[36] M. H. Fischer, "Estimating reachability: Whole body engagement or postural stability?" Human Movement Science, vol. 19, pp. 297-318, 2000.

[37] P. Rochat and M. Wraga, "An account of the systematic error in judging what is reachable." Journal of Experimental Psychology: Human Perception and Performance, vol. 23, pp. 199-212, 1997.

[38] E. Thelen and B. Ulrich, "Hidden skills: a dynamic systems analysis of treadmill stepping during the first year," Monographs of the Society for Research in Child Development, vol. 56(1), pp. 1-98, 1991.

[39] B. J. Grzyb, J. Boedecker, M. Asada, A. P. del Pobil, and L. B. Smith, "Between frustration and elation: sense of control regulates the intrinsic motivation for motor learning," in AAAI Workshop on Lifelong learning, 2011.

[40] B. J. Grzyb, A. P. del Pobil, and L. B. Smith, "How walking influences the development of absolute distance perception," in From Animals to Animats 12, ser. Lecture Notes in Computer Science, T. Ziemke, C. Balkenius, and J. Hallam, Eds. Springer Berlin / Heidelberg, 2012, vol. 7426, pp. 65-74.

[41] I. van der Fits, E. Otten, A. Klip, L. V. Eykern, and M. Hadders-Algra, "The development of postural adjustments during reaching in 6- to 18month-old infants. evidence for two transitions," Exp. Brain Res, vol. 126, pp. 517-528, 1999.

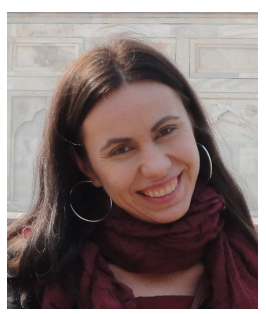

Beata J. Grzyb received her MSc Degree in Computer Science from Maria Curie-Sklodowska University in Lublin, Poland. Currently she is a $\mathrm{PhD}$ candidate in the Robotic Intelligence Lab, Jaume I University, and has already published in several journal and proceedings. In her research, she follows the approach of cognitive developmental robotics, and tackles problems related to body representation, peripersonal space representation and perception of body effectivities, by means of synthesizing neuroscience, developmental psychology, and robotics. 
This is the author's version of an article that has been published in this journal. Changes were made to this version by the publisher prior to publication.

The final version of record is available at http://dx.doi.org/10.1109/TAMD.2013.2255872

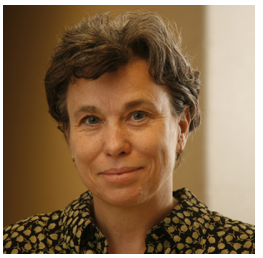

Linda B. Smith is a Distinquished Professor and the Chancellor's Professor of Psychological and Brain Sciences and Cognitive Science at Indiana University - Bloomington. She received her B.S. degree in 1973 from the University of Wisconsin - Madison and her Ph.D. in psychology from the University of Pennsylvania in 1977. She joined the faculty at Indiana University in 1977 and currently serves as Chair of the Department of Psychological and Brain Sciences. Her research is directed to understanding developmental processes especially at it applies to early cognitive development and to the interaction of perception, action and language in that developmental process. She has published over 200 research articles and is a fellow of the American Academy of Arts and Sciences, the Society of Experimental Psychologists, Cognitive Science and the American Psychological Society. She is the 2013 winner of the David E. Rumelhart Prize and was awarded the 2013 American Psychological Association Distinguished Scientific Contribution Award. You may find out more about her research and laboratory at www.iub.edu/ cogdev

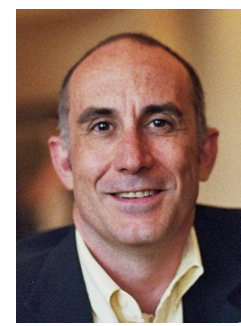

Angel P. del Pobil Angel P. del Pobil received the B.Sc. degree in physics and the Ph.D. degree in engineering from the University of Navarra, Pamplona, Spain, in 1986 and 1991, respectively. He is currently a Professor with Jaume I University (UJI), Castelln de la Plana, Spain, where he is the founding Director of the UJI Robotic Intelligence Laboratory. $\mathrm{He}$ is a World Class University Visiting Professor at Sungkyunkwan University, Seoul, Korea. He has been an Invited Speaker of over 50 tutorials, plenary talks, and seminars. He has over 230 scientific publications including eleven books. The lastest book with Mario Prats on the award-winning framework for multimodal robot physical interaction has been published in 2013 in the Springer Tracts in Advanced Robotics. His present research interests include perceptual robotics, cognitive developmental robotics, robot learning for sensorimotor interaction, and the interplay between neurobiology and robotics. Dr. del Pobil has chaired two technical committees of the IEEE Robotics and Automation Society and is a member of the Governing Board of the European Robotics Research Network (EURON) and the Intelligent Autonomous Systems (IAS) Society. He has been a Program or General Chair of six international conferences and some 18 workshops and has served on the program committees of over 115 international conferences. 